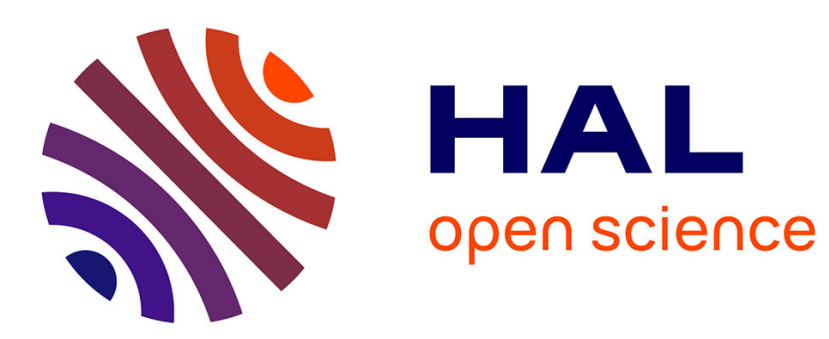

\title{
Equivalence Between Semi-empirical and Population-Balance Foam Models
}

Omar Gassara, Frédéric Douarche, Benjamin Braconnier, Bernard Bourbiaux

\section{To cite this version:}

Omar Gassara, Frédéric Douarche, Benjamin Braconnier, Bernard Bourbiaux. Equivalence Between Semi-empirical and Population-Balance Foam Models. Transport in Porous Media, 2017, 120 (3), pp.473 - 493. 10.1007/s11242-017-0935-8 . hal-01702668

\section{HAL Id: hal-01702668 \\ https://hal-ifp.archives-ouvertes.fr/hal-01702668}

Submitted on 7 Feb 2018

HAL is a multi-disciplinary open access archive for the deposit and dissemination of scientific research documents, whether they are published or not. The documents may come from teaching and research institutions in France or abroad, or from public or private research centers.
L'archive ouverte pluridisciplinaire HAL, est destinée au dépôt et à la diffusion de documents scientifiques de niveau recherche, publiés ou non, émanant des établissements d'enseignement et de recherche français ou étrangers, des laboratoires publics ou privés. 


\title{
Equivalence between semi-empirical and population-balance foam models
}

\author{
O. Gassara · F. Douarche · B. Braconnier · \\ B. Bourbiaux
}

Received: date / Accepted: date

\begin{abstract}
Models for simulating foam-based displacements in enhanced oil recovery (EOR) processes fall into two categories: population-balance (PB) models that derive explicitly foam texture, or bubble size, evolution in porous media from pore-level mechanisms related to lamellas generation and coalescence, and semi-empirical (SE) models that account implicitly for foam texture effects through a gas mobility reduction factor that depends on fluid saturation, interstitial velocity, surfactant concentration, and other factors. This mobility reduction factor has to be calibrated from a large number of experiments on a case by case basis in order to match the physical effect of each considered parameter on foam behavior. This paper develops a method for identifying the SE models from the physics of foams as derived from PB models at local equilibrium (LE). The identification of both foam flow models leads to a method for calibrating SE models from the PB model translation of foam flow data. Application to a set of foam quality-scan experiments at fixed total flow rate shows that the SE and PB models at LE match equally well the measurements and generate almost the same results in both the so-called high- and low-quality regimes. We demonstrate that the two approaches are equivalent at local equilibrium and differ only in the way in which the complex dynamic mechanisms of lamellas are handled. This physical approach of foam flow could circumvent some difficulties in the direct calibration of SE models from foam mobility (or apparent viscosity) data.
\end{abstract}

Keywords Multiphase flow · Porous media · Foam · Models · Reservoir simulation · Reservoir engineering $\cdot$ Enhanced oil recovery

O. Gassara, F. Douarche, B. Bourbiaux,

IFP Energies nouvelles, Geosciences Division, 1 et 4 avenue de Bois-Préau, 92852 Rueil-Malmaison Cedex, France,

E-mail: omar.gassara@ifpen.fr, frederic.douarche@ifpen.fr, bernard.bourbiaux@ifpen.fr

B. Braconnier,

IFP Energies nouvelles, Mechatronics and Numerics Division, 1 et 4 avenue de Bois-Préau, 92852 RueilMalmaison Cedex, France,

E-mail: benjamin.braconnier@ifpen.fr 


\section{Introduction}

In view of the recent oil production challenges resulting from rising oil demand and declining new fields discoveries, enhanced oil recovery (EOR) methods are increasingly becoming important. They follow primary production by depletion and secondary recovery methods by water or gas injection. Gas injection processes suffer from poor volumetric sweep efficiency due to both channeling and gravity override. Foam has revealed great potential to overcome these detrimental effects by reducing gas mobility, and consequently, it proves itself as a promising EOR method [1,2,3,4].

Foam consists in a succession of gas bubbles separated by thin liquid films called lamellas. Foam is usually characterized by its quality and texture. The foam quality is the gas volumetric fraction in the bulk foam, or the gas fractional rate when injected in a porous medium, and the foam texture is the number of lamellas per unit volume of foam. The gas phase flowing as a dispersed phase within a foam is much less mobile than the same gas flowing as a continuous phase. That mobility reduction can be seen as the result of an increased effective viscosity of gas, of a decreased relative permeability of gas [5], of a high trapping of gas [6], or also as a conjunction of several such phenomena. Increased effective viscosity of gas flowing as a foam can be explained thanks to a microscopic approach of foam flowing as a train of gas bubbles separated by lamellas [7], which are responsible for an additional resistance to gas flow according to Bretherton's study of bubble flow in a capillary [8]. Therefore, the effective gas viscosity in presence of foam depends strongly on foam texture: the finer the texture, the larger the number of lamellas, hence the higher resistance to the flow of the gas phase. However, foam flow properties within a porous medium are not controlled by the foam texture alone, because porous structure and pore thresholds have a determinant impact on the flow behavior of a given foam. Furthermore, foam texture can change within porous media as the result of multiple pore-level mechanisms resulting in foam bubbles creation and coalescence $[9,10,6,11]$.

Experimental evidence of lamellas coalescence in foam models led Khatib et al [10] to introduce the limiting capillary pressure $P_{c}^{*}$ concept. Above that threshold, foam films become unstable, and foam becomes coarser and coarser as a result of bubbles coalescence (cf Fig. 1). The magnitude of $P_{c}^{*}$ varies with surfactant concentration, electrolyte concentration, gas flow rate and porous medium permeability $[10,12]$.

The behavior of foam flowing at a given velocity within a porous medium exhibits two distinct flow regimes depending on foam quality [13]: a low-quality regime such that the pressure gradient, or the apparent foam viscosity, increases with foam quality until an optimal foam quality $f_{g}^{*}$ is obtained, and a high-quality regime such that the pressure gradient and the apparent foam viscosity decrease.

A variety of models has been proposed for modeling foam flow in porous media, which can be classified into two main groups. The first group includes population-balance (PB) models that account for pore-level mechanisms related to lamella creation and coalescence in order to track the dynamical behavior of foam texture in porous media along with its effect on gas mobility $[5,14,15,16]$. Alternatively, a local steady state version of PB models $[15,16,17]$ comes out by equating the lamella creation and coalescence rates; solving this equation yields the foam texture. Semi-empirical (SE) models, that constitute the second group, assume that an equilibrium state is attained instantaneously such that the foam texture effects can be represented implicitly through a gas mobility reduction factor that depends on fluid saturation, interstitial velocity, surfactant concentration and other factors $[18,19]$. SE models incorporate implicitly the limiting capillary pressure with a steep transition function which represents the coalescence effects on the mobility reduction factor. This mobility 


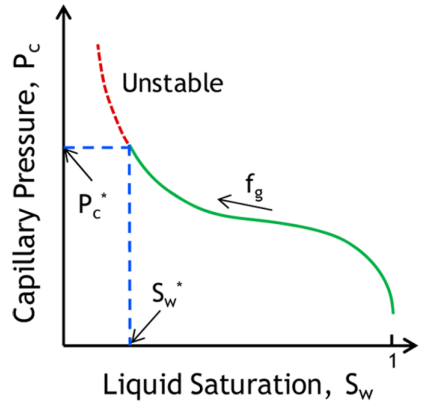

(a)

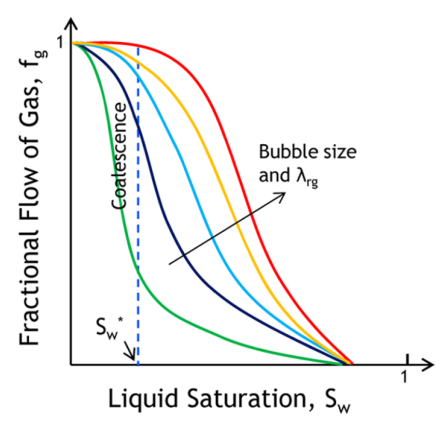

(b)

Fig. 1: Limiting capillary pressure schematics $[10,12]$. (a) Capillary pressure as a function of water saturation: capillary pressure increases with the gas saturation up to $P_{c}^{*}$, above which foam lamellas become unstable and coalesce; (b) fractional flow of gas as a function of water saturation and bubble size: when water saturation reaches $S_{w}^{*}$ corresponding to $P_{c}^{*}$, foam becomes coarser, gas fractional flow increases and consequently gas mobility increases. At low-quality regime, for $S_{w}>S_{w}^{*}$, the fractional flow of gas follows the green curve, then a transition occurs at $S_{w}=S_{w}^{*}$ (dotted line), and finally the fractional flow of gas follows the red curve at high-quality regime for $S_{w}<S_{w}^{*}$.

reduction factor has to be calibrated from a large number of experiments on a case by case basis in order to match the physical effect of each considered parameter on foam behavior.

In the laboratory, the foam flow behavior in a porous medium is generally characterized by its apparent viscosity $\mu_{f}^{\text {app }}$. Considering foam as a single homogeneous phase in a porous medium whose boundaries are subjected to a pressure difference $\Delta P$, one can apply Darcy law and define the foam apparent viscosity as $\mu_{f}^{\text {app }}=\frac{k}{|\mathbf{u}|} \frac{\Delta P}{L}$, where $k$ is the average porous media permeability, $L$ the porous medium length across which the pressure difference is applied, and $|\mathbf{u}|$ the foam velocity. Procedures to fit SE models to foam quality-scan experiments at fixed total flow rate have been successfully provided by several authors $[19,20,21$, 12]. They are discussed later on, and compared to the present paper method.

This paper develops a method for identifying the SE models from the physics of foams as derived from PB models. The motivation of this work is to strengthen further the physical basis of SE models and constrain the model calibration by reproducing as closely as possible the foam texture effects on gas mobility, as suggested by PB models. The addressed items are covered in the following, namely:

- Summary of PB and SE models features and differences,

- Equivalence between SE and PB models at local equilibrium,

- Validation of the proposed SE models identification method against experimental data,

- Comparison between the results of this work with others found in the literature.

\section{Foam modeling approaches}

In this section, we detail the two main modeling categories that describe foam in the framework of a reservoir simulator, namely the population-balance (PB) models and the semiempirical (SE) models. These models extend the usual three phase reservoir simulators 
based on the generalized Darcy law. Thus, for these models, foam is not modeled as an additional phase: rather, it is still described as a mixture of two water and gas phases, where the gas phase mobility is modified and the liquid phase mobility remains unchanged [22, $23,14]$. In addition, both models require to model a surfactant which is needed for the foam or lamellas formation and stability. Before underlining PB and SE models specific features regarding the gas mobility, we present the material balance equations for the water, hydrocarbon and surfactant components that underlie both types of model.

\subsection{Hydrocarbons, surfactant and water mass balance equations}

We consider a model for a three-phase flow in a porous medium in the presence of foam. We distinguish three phases: an aqueous phase $w$, an oil phase $o$ and a gas phase $g$. This flow is modified by the presence of foam. Modeling foam requires the presence of a surfactant, which is transported by the water phase, and which is described by an additional mass balance equation. The surfactant is either mobile or adsorbed on the rock. When present in the water phase, we assume that the surfactant mass does not affect the water mass.

It has been shown $[22,23,14]$ that the transport of liquid is not affected by the presence of foam. On the opposite, the gas velocity is significantly reduced by the presence of foam. Thus, to describe the water and hydrocarbon phases, we consider a black-oil model [24, $25]$ where the gas phase involves a modified velocity which will be denoted $\mathbf{u}_{g}^{f}$. The mass conservation equations read

$$
\left\{\begin{array}{l}
\partial_{t}\left(\Phi \rho_{w} S_{w}\right)+\nabla \cdot\left(\rho_{w} \mathbf{u}_{w}\right)=q_{w}, \\
\partial_{t}\left(\Phi \rho_{w} S_{w} C_{w}^{s}+(1-\Phi) \rho_{r} C_{r}^{s}\right)+\nabla \cdot\left(\rho_{w} \mathbf{u}_{w} C_{w}^{s}\right)=q_{w} C_{w}^{s}, \\
\partial_{t}\left(\Phi C_{h} \rho_{o} S_{o}\right)+\nabla \cdot\left(\rho_{o} C_{h} \mathbf{u}_{o}\right)=C_{h} q_{o}, \\
\partial_{t}\left(\Phi \rho_{g} S_{g}+\Phi C_{v} \rho_{o} S_{o}\right)+\nabla \cdot\left(\rho_{o} C_{v} \mathbf{u}_{o}+\rho_{g} \mathbf{u}_{g}^{f}\right)=q_{g}+C_{v} q_{o}
\end{array}\right.
$$

where $\Phi$ is the rock porosity. For each phase denoted $\psi=w, o, g, S_{\psi}$ is the saturation, $\rho_{\psi}$ the mass density and $q_{\psi}$ the source/sink term per unit volume of porous medium. The gas phase contains a single volatile component denoted $v$, whereas the oil phase contains a heavy component denoted $h$ and previous volatile component $v$, with $C_{h}$ and $C_{v}=1-C_{h}$ their respective mass fraction (in that phase). The equilibrium constant $K_{v}=\frac{1}{X_{v}}$, with $X_{v}$ the molar fraction of volatile component in the oil phase, is defined from $C_{v}$ and $C_{h} . K_{v}$ is a function of pressure. $C_{w}^{s}$ stands for the flowing surfactant mass fraction in the water phase and $C_{r}^{s}$ for the adsorbed surfactant mass fraction on the rock with $\rho_{r}$ the rock mass density. Mobile and adsorbed surfactant mass fractions are related with an adsorption law such as the Langmuir isotherm [26,27].

Under creeping (i.e. low-velocity) flow conditions, the pure phase velocities in permeable porous media are governed by the generalized Darcy law:

$$
\mathbf{u}_{\psi}=-\frac{k k_{r \psi}}{\mu_{\psi}}\left(\nabla P_{\psi}-\rho_{\psi} \mathbf{g}\right),
$$

where $k$ is the rock permeability, $\mu_{\psi}$ the pure phase viscosity, $P_{\psi}$ the pressure of the phase $\psi$ and $\mathbf{g}$ the gravity. $k_{r \psi}$ is the relative permeability for the pure phase $\psi$, i.e. without lamellas. We suppose the relative permeabilities and capillary pressure functions are known, with given saturation end points. In our specific context of foam modeling, we will only consider the gas and water phases, thus $S_{g} \in\left[S_{g r}, 1-S_{w i}\right]$ where $S_{g r}$ is the residual gas saturation and $S_{w i}$ the irreducible water saturation. 
In order to simplify the notations in the following, we introduce the phase mobility $\lambda_{\psi}=$ $k_{r \psi} / \mu_{\psi}$ and the interstitial velocity $\mathbf{v}_{\psi}=\mathbf{u}_{\psi} /\left(\Phi S_{\psi}\right)$ (see [28] for more details). We also introduce the phase fractional flow $f_{\psi}=\left|\mathbf{u}_{\psi}\right| /|\mathbf{u}|$ where the total velocity $\mathbf{u}=\sum_{\psi \in\{w, o, g\}} \mathbf{u}_{\psi}$ is the sum of the phase velocities.

The flow of gas in the presence of foam is discussed later on as it is modeled differently whether a population-balance or an semi-empirical modeling approach is used. These models are based on Darcy type laws which are extended to obtain a modified gas velocity so that

$$
\mathbf{u}_{g}^{f}= \begin{cases}\mathbf{u}_{g}^{\mathrm{PB}} & \text { for PB models, } \\ \mathbf{u}_{g}^{\mathrm{SE}} & \text { for SE models, }\end{cases}
$$

where $\mathbf{u}_{g}^{\mathrm{PB}}$ stands for the gas velocity in the presence of foam according to PB models and $\mathbf{u}_{g}^{\mathrm{SE}}$ is the gas velocity in the presence of foam for the SE models. These two gas velocities models are detailed in the two next sections.

\subsection{Population-balance lamellas models}

Foam mobility is strongly related to its texture which is a key variable in foam modeling in porous media: as foam texture increases, the resistance to gas flow in porous media increases. Population-balance models were designed in order to relate explicitly the gas mobility reduction to the foam texture. Thus, for PB models, the dynamics of foam texture needs to be modeled and a lamella population-balance equation is considered. The impact of foam texture on gas mobility is modeled through an effective gas viscosity and a gas trapping for more elaborated models $[14,17,6]$. In this study, we focus on viscosity-type PB models and do not consider gas trapping.

The population-balance model involves a lamellas balance that includes lamellas advection at the modified gas velocity and source/sink terms taking into account lamellas creation and destruction $[29,5]$. The lamellas population balance equation reads

$$
\partial_{t}\left(\Phi S_{g} n_{f}\right)+\nabla \cdot\left(\mathbf{u}_{g}^{\mathrm{PB}} n_{f}\right)=\Phi S_{g}\left(r_{g}-r_{c}\right)+q_{f},
$$

where $n_{f}$ is the number of flowing lamellas (or foam bubbles) per unit volume of gas and $q_{f}$ is the external lamellas source/sink term (number of lamellas per unit of time).

As mentioned earlier, the gas velocity is significantly reduced when foam develops. The viscosity-type PB models extend the generalized Darcy equation for the gas phase as follows:

$$
\mathbf{u}_{g}^{\mathrm{PB}}=-\frac{k k_{r g}}{\mu_{g}^{\mathrm{PB}}}\left(\nabla P_{g}-\rho_{g} \mathbf{g}\right),
$$

where $\mu_{g}^{\mathrm{PB}}$ is the effective gas viscosity when flowing as a succession of bubbles. The rheology of PB models of foam flow is classically based on Bretherton's flow model of a single bubble within a capillary [8], that was later extended by Hirasaki and co-workers to a train of bubbles [7] thus leading to the following expression for the effective gas viscosity:

$$
\mu_{g}^{\mathrm{PB}}=\mu_{g}+\frac{c_{f} n_{f}}{\left|\mathbf{v}_{g}^{\mathrm{PB}}\right|^{1 / 3}}
$$

where $\mathbf{v}_{g}^{\mathrm{PB}}$ is the interstitial gas velocity in the presence of foam, deduced from $\mathbf{u}_{g}^{\mathrm{PB}} \cdot c_{f}$ is a constant depending on the surfactant concentration and the permeability of the porous medium [16]. $r_{g}$ and $r_{c}$ are the rates of lamellas creation and coalescence. 
Different formulas have been proposed in the PB models of the literature to model these rates $[6,30,31]$. For the purpose of our paper, we propose to focus on the Kam et al [30] model for which the creation and coalescence rates read:

$$
r_{g}=c_{g} S_{w}\left|\nabla P_{g}\right|^{m}, \quad \quad r_{c}=\frac{c_{c} n_{f}}{\left(S_{w}-S_{w}^{*-\mathrm{PB}}\right)^{n}},
$$

where $c_{g}$ is the generation rate coefficient, $c_{c}$ the coalescence rate coefficient, $n$ a coalescence exponent, $m$ a model parameter, and where the foam texture $n_{f}$ cannot exceed a maximum value $n_{f}^{\max }$.

The formulations of these rates need to be commented as they involve porous medium control on the typical foam behavior that can be observed in core flow experiments. Regarding lamellas generation, Kam et al [30] suggested that the rate of lamellas creation in porous media is proportional to the water saturation and to a power-law expression of the gas-phase pressure gradient. Specifically, foam generation is easier at higher water saturation because more liquid lenses can be generated within the pore network. Regarding lamellas coalescence, the rate $r_{c}$ is expressed as a function of the foam texture $n_{f}$ and the limiting water saturation $S_{w}^{*-\mathrm{PB}}$, corresponding to the limiting capillary pressure $P_{c}^{*}$. The expression of $r_{c}$ predicts that lamellas destruction diverges toward infinity as $S_{w}$ approaches $S_{w}^{*-\mathrm{PB}}$.

From a more mathematical point of view, let us observe that the local steady state version of the population-balance model is obtained by assuming local steady state and instantaneous equilibrium between the rates of foam generation and coalescence. This equality between $r_{g}$ and $r_{c}$ yields the foam texture, as will be shown in Section 4.

As regards the foam texture in a porous medium, it is acknowledged that the size of foam bubbles (that is the inverse of $n_{f}$ ) is closely depending on the porous structure [5,14]. In first approximation, it can be estimated as the volume of spherical bubbles with a radius $r$ in the order of characteristic pore radius, the latter being a function of porous medium flow properties [16]. Following Alvarez et al's working hypothesis [32], Kam et al [30] assume that the size of individual foam bubbles is constant in the low-quality regime once the conditions of foam generation (velocity) are met, then increases in the high-quality regime because of coalescence. To end with, according to Kam et al model, the maximum foam texture $n_{f}^{\max }$ is obtained in the low-quality regime and can be estimated from a characteristic pore radius. However, that question of foam texture remains controversial because the mechanisms of lamellas generation are complex [6] and hardly tractable in the context of natural porous media, but such a discussion is beyond the scope of present work.

Overall, expressions (6) incorporate the qualitative physical trends of foam texture dependence on the porous medium as discussed above, but they still have to be calibrated from experiments, such as the ones from Alvarez et al [32] and Moradi-Araghi et al [33].

\subsection{Semi-empirical foam models}

Most semi-empirical (SE) foam flow simulators are based on the same modeling approach that consists in applying a gas mobility reduction factor when foam is present. A SE model implemented in IFPEN simulator PumaFlow [34] is considered herein for the only purpose of demonstrating the identification methodology of that paper. Herein, a two-phase gaswater fluid system is considered. Surfactant is transported by the water phase and assumed 
not to adsorb on the rock. Hence, the mass conservation equations system (1) reads

$$
\left\{\begin{array}{l}
\partial_{t}\left(\Phi \rho_{w} S_{w}\right)+\nabla \cdot\left(\rho_{w} \mathbf{u}_{w}\right)=q_{w}, \\
\partial_{t}\left(\Phi \rho_{w} S_{w} C_{w}^{s}\right)+\nabla \cdot\left(\rho_{w} \mathbf{u}_{w} C_{w}^{s}\right)=q_{w} C_{w}^{s}, \\
\partial_{t}\left(\Phi \rho_{g} S_{g}\right)+\nabla \cdot\left(\rho_{g} \mathbf{u}_{g}^{f}\right)=q_{g} .
\end{array}\right.
$$

The main features of that SE model are described hereafter. The gas mobility $\lambda_{g}^{\mathrm{SE}}$ of SE model is scaled by a multi-parameter interpolation function $F M$ assigned to the relative permeability while the gas viscosity is assumed unchanged whether foam is present or not:

$$
\mathbf{u}_{g}^{\mathrm{SE}}=-k \lambda_{g}^{\mathrm{SE}}\left(\nabla P_{g}-\rho_{g} \mathbf{g}\right) \quad \text { with } \quad \lambda_{g}^{\mathrm{SE}}=\frac{k_{r g}^{\mathrm{SE}}}{\mu_{g}}=\frac{F M \cdot k_{r g}}{\mu_{g}},
$$

where $k_{r g}^{\mathrm{SE}}$ is the modified gas relative permeability for the SE model in the presence of foam and $k_{r g}$ is the conventional relative permeability of gas. $F M$ is a multi-parameter interpolation functional form that includes the contributions of physical parameters impacting the gas mobility reduction. FM is formulated as follows:

$$
F M=\frac{1}{1+\left(M_{\mathrm{ref}}-1\right) \prod_{i=1}^{4} F_{i}},
$$

where $M_{\text {ref }}$ is the reference (maximum) gas mobility reduction under optimal conditions of the rock-fluid-additive system under consideration, and $F_{i}$ are functions of four physical parameters that are surfactant concentration, water saturation, oil saturation, and gas velocity, or equivalently, the gas capillary number. In this paper, we study the effect of water saturation and gas velocity on foam performance. Thus, we focus on the so called dry-out function $F_{2}$ and shear-thinning function $F_{4}\left(F_{1}\right.$ and $F_{3}$ functions account for surfactant concentration and oil saturation effect on foam performance, and are not developed herein). In our model, $F_{2}$ reads

$$
F_{2}\left(S_{w}\right)=\frac{1}{2}+\frac{1}{\pi} \arctan \left[\Theta\left(S_{w}-S_{w}^{*-S E}\right)\right],
$$

where the dimensionless constant $\Theta$ governs the sharpness of the transition from the lowquality regime to the high-quality regime when water saturation decreases below $S_{w}^{*-\mathrm{SE}}$. A very high value of $\Theta$ (several thousands) leads to a steep transition and in this case foam coalescence occurs at the given water saturation $S_{w}^{*-S E}$, whereas a low value (less than 100 or 10) means a smooth transition in the vicinity of $S_{w}^{*-\mathrm{SE}}$.

Furthermore, $F M$ includes a shear-thinning function $F_{4}$ expressing the non-Newtonian behavior of foam flow in porous media: at fixed foam parameters (surfactant concentration, foam texture and fluids saturations), foam viscosity decreases with gas velocity, and subsequently, foam mobility increases $[8,7,5,14]$. In SE models, this dependence is expressed via the local gas capillary number $N_{c g}$ which represents the relative effect of viscous forces versus capillary ones. The shear-thinning function is defined as

$$
F_{4}\left(N_{c g}\right)=\left(\frac{N_{c g}^{\mathrm{ref}}}{N_{c g}}\right)^{e_{c}} \quad \text { with } \quad N_{c g}=\frac{\mu_{g}\left|\mathbf{u}_{g}^{\mathrm{SE}}\right|}{\Phi \sigma S_{g}}
$$

where $N_{c g}^{\text {ref }}$ is a reference capillary number for which $M_{\text {ref }}$ is measured, $e_{c}$ an exponent representing the extent of shear-thinning behavior and $\sigma$ the interfacial tension between gas and water. Shear-thinning behavior can be ignored by setting $e_{c}=0$. 


\section{Identification of SE and PB models at local steady state}

As only local steady state foam flow is modeled by SE model, the identification procedure developed hereafter will involve only the solutions of PB models at local equilibrium. As explained in the previous section, the gas-phase mobility $\lambda_{g}^{\mathrm{PB}}$ in most PB models is based on a modified gas viscosity whereas the foam mobility $\lambda_{g}^{\mathrm{SE}}$ in SE models is based on a modified relative permeability of gas. Hence, the identification of SE-model gas mobility to PB-model gas mobility is $\lambda_{g}^{\mathrm{SE}}=\lambda_{g}^{\mathrm{PB}}$, that is $k_{r g}^{\mathrm{SE}} / \mu_{g}=k_{r g} / \mu_{g}^{\mathrm{PB}}$. Reintroducing the expressions of $k_{r g}^{\mathrm{SE}}$ and $\mu_{g}^{\mathrm{PB}}$ given by Eqs (8), (9) and (5) we obtain:

$$
\left(M_{\mathrm{ref}}-1\right) \prod_{i=1}^{4} F_{i}=\frac{c_{f} n_{f}}{\mu_{g}\left|\mathbf{v}_{g}^{\mathrm{PB}}\right|^{1 / 3}} .
$$

Assuming no oil and optimal concentration of foaming additive, the $\prod_{i=1}^{4} F_{i}$ product only involves the dry-out and shear thinning functions $F_{2}$ and $F_{4}$ given by Eqs (10) and (11). In order to go further with the identification, the interstitial velocity is expressed as a function of the capillary number $N_{c g}$ defined in Eq. (11), such that Eq. (12) can be rewritten as:

$$
\left(M_{\mathrm{ref}}-1\right) F_{2}\left(\frac{N_{c g}^{\mathrm{ref}}}{N_{c g}}\right)^{e_{c}}=\frac{c_{f} n_{f}}{\mu_{g}}\left(\frac{\mu_{g}}{N_{c g} \sigma}\right)^{1 / 3} .
$$

Rearranging shows that the right member emanating from PB model includes the $N_{c g}$ dependent $F_{4}$ function corresponding to the shear-thinning regime:

$$
\left(M_{\mathrm{ref}}-1\right) F_{2}\left(\frac{N_{c g}^{\mathrm{ref}}}{N_{c g}}\right)^{e_{c}}=\frac{c_{f} n_{f}}{\mu_{g}}\left(\frac{\mu_{g}}{N_{c g}^{\mathrm{ref}} \sigma}\right)^{1 / 3}\left(\frac{N_{c g}^{\mathrm{ref}}}{N_{c g}}\right)^{1 / 3} .
$$

At that stage, a first identification item can be pointed out, that concerns the shearthinning impact on the mobility reduction function. Previous equation shows that dependence on velocity, here expressed through $N_{c g}$, involves an exponent $e_{c}$ equal to $\frac{1}{3}$ according to the motion law of bubbles established by Bretherton [8]. We note that both our SE model and under consideration PB model account for the shear-thinning effect on foam performance for any gas velocity, because the latter is assumed higher than a minimum value corresponding to $N_{c g}^{\text {ref }}$ and satisfying foam generation.

To go further on with $F_{2}$ identification, we note that, apart from the velocity-dependent $N_{c g}$ term, on the one hand the remaining variable is $n_{f}$, and on the other hand $F_{2}$ is a function that is scaled between 0 and 1 . Therefore, $F_{2}$ can be identified as follows

$$
F_{2}=A n_{f},
$$

where $A$ is a constant. Allowing $F_{2}$ to reach the unity value requires to set $A$ to the inverse of the maximum foam texture $n_{f}^{\max }$. It is worth underlining that the shear-thinning mechanism is ignored in $F_{2}$ identification, because that mechanism is held by $N_{c g}$-dependent function.

The dry-out function $F_{2}$ models the decreasing impact of foam on mobility when the porous medium induces a coalescence of foam bubbles because of capillary pressure effects on foam films stability $[12,21,20]$. In other words, the volumetric concentration of bubbles, i.e. the foam texture $n_{f}$, decreases rapidly as gas saturation exceeds a certain threshold value that limits films stability. However, that coalescence of the foam occurs over a certain range of capillary pressure values (or of films disjoining pressures). Such a behavior is well represented by the $F_{2}$ function given by Eq. (10), $F_{2}\left(S_{w}\right)=\frac{1}{2}+\frac{1}{\pi} \arctan \left[\Theta\left(S_{w}-S_{w}^{*-\mathrm{SE}}\right)\right]$, where 
the $S_{w}^{*-\mathrm{SE}}$ saturation corresponds to the middle of the saturation range $\left[S_{w}^{*-\mathrm{SE}}-\delta, S_{w}^{*-\mathrm{SE}}+\delta\right]$ over which foam collapses and the $\Theta$ parameter drives the width of that saturation range.

The $F_{2}$ function accounts directly for the dependence of foam performance on the porous medium saturation whereas in PB models at local steady state, the same dependence is modeled indirectly via the foam texture that is itself modeled as a function of saturation. Considering the Kam's model taken as PB model type, the threshold saturation $S_{w}^{*-\mathrm{PB}}$ involved in that PB model is analog but not exactly equal to the water saturation threshold $S_{w}^{*-S E}$ of the SE model, because $S_{w}^{*-\mathrm{PB}}$ is the lowest water saturation value below which foam no more exists, which corresponds to a value of the $F_{2}$ function equal to 0 . For the sake of clarity, we will then keep on distinguishing $S_{w}^{*-\mathrm{SE}}$ and $S_{w}^{*-\mathrm{PB}}$, whose difference is given by $\delta=S_{w}^{*-\mathrm{SE}}-S_{w}^{*-\mathrm{PB}}=F_{2}^{-1}\left(\frac{1}{2}\right)-F_{2}^{-1}(0)$ where $F_{2}^{-1}$ is the inverse of the $F_{2}\left(S_{w}\right)$ function.

Obviously, regarding the $F_{2}$ function, the identification of SE model to PB model on the basis of their respective gas mobilities at local steady state as written before in Eq. (14) does not help to determine directly $\Theta$ and $S_{w}^{*-S E}$ as an expression of PB model parameters. Thus, $F_{2}$ can only be identified globally as the dimensionless foam texture $n_{f} / n_{f}^{\max }$, knowing however that all the underlying physics of porous medium effects as described before is held in the foam texture, that is the local steady state solution of the PB model. That is, we can write

$$
F_{2}=\frac{n_{f}}{n_{f}^{\max }}=n_{D}
$$

where $n_{D}$ is the dimensionless foam texture. Different values of the foam texture $n_{f}^{i}$ and of the corresponding saturation $S_{w}^{i}$ can be determined by solving PB model at local steady state for a set of foam quality values $f_{g}^{i}$, as explained in Section 4 . That is, $\Theta$ and $S_{w}^{*-S E}$ constants can be determined through an optimization of a set equations written as

$$
\frac{n_{f}^{i}\left(S_{w}^{i}\right)}{n_{f}^{\max }}=\frac{1}{2}+\frac{1}{\pi} \arctan \left[\Theta\left(S_{w}^{i}-S_{w}^{*-\mathrm{SE}}\right)\right] .
$$

Alternatively, one can determine directly the limiting water saturation $S_{w}^{*-S E}$ in SE models from the dimensionless foam texture function $n_{D}$ as $S_{w}^{*-S E}=n_{D}^{-1}\left(\frac{1}{2}\right)$, where $n_{D}^{-1}$ is the inverse of the $n_{D}\left(S_{w}\right)$ function. Once $S_{w}^{*-\mathrm{SE}}$ is calculated, the parameter $\Theta$ can be determined by setting the $F_{2}$ function to zero at $S_{w}^{*-\mathrm{PB}}$, i.e. $F_{2}\left(S_{w}^{*-\mathrm{PB}}\right)=0$. As such a value cannot be strictly attained, a tolerance $\varepsilon$ should be permitted on $F_{2}\left(S_{w}^{*-\mathrm{PB}}\right)$ such that $\frac{1}{2}+$ $\frac{1}{\pi} \arctan \left[\Theta\left(S_{w}^{*-\mathrm{PB}}-S_{w}^{*-\mathrm{SE}}\right)\right]=\varepsilon$, that can also be written as $\frac{1}{2}-\frac{1}{\pi} \arctan (\Theta \delta)=\varepsilon$, which yields $\Theta=\frac{1}{\delta} \tan \left[\pi\left(\frac{1}{2}-\varepsilon\right)\right]$. The choice of tolerance $\varepsilon$ is discussed later on in Section 5.3.

To end up with the identification of SE model empirical parameters to PB model, we rewrite Eq. (14) as

$$
\left(M_{\mathrm{ref}}-1\right) F_{2}\left(\frac{N_{c g}^{\mathrm{ref}}}{N_{c g}}\right)^{e_{c}}=\frac{c_{f} n_{f}^{\max }}{\mu_{g}}\left(\frac{\mu_{g}}{N_{c g}^{\mathrm{ref}} \sigma}\right)^{1 / 3} n_{D}\left(\frac{N_{c g}^{\mathrm{ref}}}{N_{c g}}\right)^{1 / 3}
$$

Taking into account previous identifications, $M_{\text {ref }}$ expression versus PB model parameters is

$$
M_{\mathrm{ref}}=1+\frac{c_{f} n_{f}^{\max }}{\mu_{g}}\left(\frac{\mu_{g}}{N_{c g}^{\mathrm{ref}} \sigma}\right)^{1 / 3}
$$


Identification summary is then:

$$
M_{\mathrm{ref}}=1+\frac{c_{f} n_{f}^{\max }}{\mu_{g}}\left(\frac{\mu_{g}}{N_{c g}^{\mathrm{ref}} \sigma}\right)^{1 / 3}, \quad F_{2}=\frac{n_{f}}{n_{f}^{\max }}=n_{D}, \quad F_{4}=\left(\frac{N_{c g}^{\mathrm{ref}}}{N_{c g}}\right)^{1 / 3} .
$$

To proceed with the identification of an SE model using the results of the above identification, we need to know the foam texture $n_{f}$ for various foam flow conditions. This is provided by the solution of PB models at local steady state which is detailed hereafter.

\section{Analytical approach to relate gas saturation and foam texture at local steady state}

In this section, we propose an analytical approach to calculate foam texture at local steady state. The texture is obtained by equating the generation and coalescence rates of the PB model given by Eq. (6). The basic idea consists in solving jointly the foam-gas fractional flow equation and the local equilibrium equation, with gas saturation and foam texture as unknowns. Indeed, the foam-gas fractional flow $f_{g}$ is a known parameter of the problem at local steady state. The problem set-up is worth being detailed due to the peculiarity of entailed resolution.

Herein, a two-phase gas-water fluid system is considered. Capillary pressure and gravity are neglected. Porosity is assumed constant. A one-dimensional incompressible flow is considered without any sink terms. The PB model equations (1) and (4) can be combined into the following fractional flow equation

$$
\partial_{t}\left(S_{g}\right)+\frac{u}{\Phi} \partial_{x}\left(f_{g}\left(S_{g}, n_{f}\right)\right)=0,
$$

where, for the PB model, the gas fractional flow rate depends on gas saturation and foam texture:

$$
f_{g}\left(S_{g}, n_{f}\right)=\frac{1}{1+\left(\mu_{g}+\frac{c_{f} n_{f}}{\left(v_{g}^{\mathrm{PB}}\right)^{1 / 3}}\right) \frac{k_{r w}}{\mu_{w} k_{r g}}} .
$$

That equation can be inverted in order to express the foam texture as a function of saturation and gas fractional flow, that is, denoting $n_{f}^{\mathrm{FF}}$ this fractional-flow-derived foam texture:

$$
n_{f}^{\mathrm{FF}}=\left(\frac{u}{\Phi S_{g}}\right)^{1 / 3} \frac{1-f_{g}\left(1+\frac{\mu_{g}}{\mu_{w}} \frac{k_{r w}}{k_{r g}}\right)}{f_{g}^{2 / 3} \frac{c_{f}}{\mu_{w}} \frac{k_{r w}}{k_{r g}}} .
$$

At local equilibrium, the generation and coalescence rates of the PB model are equal. According to Eq. (6) and considering the maximum texture limitation, this local equilibrium yields the following expression of the foam texture at local equilibrium denoted $n_{f}^{\mathrm{LE}}$ :

$$
n_{f}^{\mathrm{LE}}= \begin{cases}\frac{c_{g}}{c_{c}} S_{w}\left(S_{w}-S_{w}^{*-\mathrm{PB}}\right)^{n}|\nabla P|^{m} & \text { if } n_{f}<n_{f}^{\max } \\ n_{f}^{\max } & \text { otherwise }\end{cases}
$$

where $\nabla P$ is the pressure gradient which can be expressed from Darcy equation applied to the water phase, whose flow properties are not affected by the presence of foam:

$$
|\nabla P|=\frac{\mu_{w} u\left(1-f_{g}\right)}{k k_{r w}} .
$$


As explained in Section 2.2, the maximum foam texture $n_{f}^{\max }$ is estimated as the characteristic pore size of the porous medium under consideration. This is an approximation for natural porous media characterized by a more or less complex pore size distribution. Therefore, it remains a variable subject to adjustment in PB models at local equilibrium [17].

Following the expression of foam texture at local equilibrium given by Eq. (24), problem solving should distinguish two cases:

- For any $f_{g}$ within the low-quality regime, foam texture is constant and is equal to the maximum value $\left(n_{f}=n_{f}^{\max }\right)$. Thus, only gas saturation remains to be calculated from the gas fractional flow equation (23), which yields

$$
S_{g}=\frac{u}{\Phi}\left[\frac{1-f_{g}\left(1+\frac{\mu_{g}}{\mu_{w}} \frac{k_{r w}}{k_{r g}}\right)}{n_{f}^{\max } f_{g}^{2 / 3} \frac{c_{f}}{\mu_{w}} \frac{k_{r w}}{k_{r g}}}\right]^{3} .
$$

- For any given $f_{g}$ within the high-quality regime $\left(n_{f}<n_{f}^{\max }\right)$, equating Eqs (24) and (23) yields the following gas saturation equation:

$$
\overbrace{\frac{c_{g}}{c_{c}}\left(1-S_{g}\right)\left(1-S_{g}-S_{w}^{*-\mathrm{PB}}\right)^{n}\left|\frac{\mu_{w} u\left(1-f_{g}\right)}{k k_{r w}}\right|^{m}}^{n_{f}^{\mathrm{LE}}\left(S_{g}\right)}=\overbrace{\left(\frac{u}{\Phi S_{g}}\right)^{1 / 3} \frac{1-f_{g}\left(1+\frac{\mu_{g}}{\mu_{w}} \frac{k_{r w}}{k_{r g}}\right)}{f_{g}^{2 / 3} \frac{c_{f}}{\mu_{w}} \frac{k_{r w}}{k_{r g}}}}^{n_{f}^{\mathrm{FF}}\left(S_{g}\right)}
$$

where $n_{f}^{\mathrm{LE}}$ represents the foam texture obtained from the local equilibrium of the PB model and $n_{f}^{\mathrm{FF}}$ is the foam texture computed from the gas fractional flow equation in the presence of foam.

Equations (26) and (27) have to be solved with numerical methods, as described later on in Section 5.

Finally, a series of measurements varying the foam quality at fixed total injection rate is needed in order to relate the gas saturation to the foam texture at local equilibrium for any quality of the foam under consideration. This relationship underlies the possibility to identify an SE model to a PB model.

\section{Application to a foam data set: identification of our SE model to calibrated Kam et al PB model}

We apply the previous identification method to calibrate our SE model to a given experimental data set published by Alvarez et al [32]. Specifically, the authors measured the steady state apparent foam viscosity for several foam qualities at constant total injection rate. All these experiments are two-phase flow displacements involving water, gas and surfactant.

The considered PB model for the application of the calibration method is previouslydescribed in the Section 2.2. We focus on the steady state foam flow that is representative of flow conditions achieved in a reservoir beyond the near-wellbore region where foam generation conditions are fulfilled. 
5.1 Case study: Alvarez et al experiments [32]

The experiments of Alvarez et al [32] are conducted on a Berea sandstone core with a $\mathrm{N}_{2}$ based foam at ambient temperature and an outlet pressure of 41 bar. The core is $5 \mathrm{~cm}$ in diameter and $28 \mathrm{~cm}$ in length. The aqueous phase of foam is a $1 \mathrm{wt} \%$ solution of surfactant in a brine containing $3 \mathrm{wt} \% \mathrm{NaCl}$ and $0.01 \mathrm{wt} \% \mathrm{CaCl}_{2}$.

A pre-generated foam of given quality is injected in the water-saturated core. While keeping constant the total flow rate of gas and water, the foam quality is changed from one experiment to another to cover a range from 0.25 to 0.91 . The measured apparent foam viscosity ranges from 274 to $905 \mathrm{cP}$. The transition between the two regimes occurs at the optimal foam quality $f_{g}^{*}=0.78$ for which the maximum apparent foam viscosity of $905 \mathrm{cP}$ is reached, as reported in Fig. 4. Table 1 summarizes the rock and fluids properties of these foam displacement experiments.

Recently, Lotfollahi et al [35] performed an extensive treatment of the foam apparent viscosity data recorded during these experiments. They calibrated different SE models, as well as three PB models, among which Kam et al model [30]. Specifically, adjustable parameters were $c_{g} / c_{c}, c_{f}, n, m$ and $S_{w}^{*-\mathrm{PB}}$. The adjusted parameter values that fit the experimental data are reported in Table 2 (gas and water relative permeabilities in the absence of foam were adjusted with power laws, namely $k_{r w}=k_{r w}^{0} S^{n_{w}}$ and $k_{r g}=k_{r g}^{0}(1-S)^{n_{g}}$ with $S=\frac{S_{w}-S_{w i}}{1-S_{g r}-S_{w i}}$.

Table 1: Rock and fluids properties of Alvarez et al foam displacements [32].

\begin{tabular}{ll|ll}
\hline Gas & $\mathrm{N}_{2}$ & $u(\mathrm{~cm} /$ hour $)$ & 3.175 \\
$T\left({ }^{\circ} \mathrm{C}\right)$ & 25 & $f_{g}$ range $(-)$ & $0.25-0.91$ \\
$P(\mathrm{bar})$ & 41 & $\mu_{w}(T, P)(\mathrm{cP})$ & 0.7 \\
$\sigma(\mathrm{mN} / \mathrm{m})$ & 30 & $\mu_{g}(T, P)(\mathrm{cP})$ & 0.02 \\
$k(\mathrm{mD})$ & 530 & $\rho_{w}(T, P)\left(\mathrm{kg} / \mathrm{m}^{3}\right)$ & 998 \\
$\Phi(-)$ & 0.18 & $\rho_{g}(T, P)\left(\mathrm{kg} / \mathrm{m}^{3}\right)$ & 46 \\
\hline
\end{tabular}

Table 2: Kam et al model (2007) parameters adjusted on Alvarez et al experiments [32] by Lotfollahi et al [35].

\begin{tabular}{ll|ll}
\hline Gas and water relative permeabilities & & Kam et al model parameters & \\
\hline$S_{w i}(-)$ & 0.2 & $S_{w}^{*-\mathrm{PB}}(-)$ & 0.31 \\
$S_{g r}(-)$ & 0.2 & $n_{f}^{\max }\left(\mathrm{m}^{-3}\right)$ & $19.1 \times 10^{12}$ \\
$k_{r w}^{0}(-)$ & 0.2 & $c_{f}\left(\mathrm{~m}^{10 / 3} \mathrm{hour}^{-1 / 3} \mathrm{cP}\right)$ & $2.77 \times 10^{-11}$ \\
$k_{r g}^{0}(-)$ & 0.94 & $c_{g} / c_{c}(-)$ & $8 \times 10^{16}$ \\
$n_{w}(-)$ & 4.2 & $n(-)$ & 1.91 \\
$n_{g}(-)$ & 1.3 & $m(-)$ & 0.2 \\
\hline
\end{tabular}

In the following section, we first compute the local steady state foam texture using the Kam et al model parameters given in Table 2, since it is the first step for SE model identification to PB model according to Eq. (20). 
5.2 Determination of foam texture at local equilibrium using Kam et al PB model: a posteriori validation

As explained in Section 4, one can solve Kam et al model at local equilibrium by equating Eqs (24) and (23). Accordingly, two regimes should be distinguished for foam texture calculations. On the one hand, within the low-quality regime, the foam texture $n_{f}$ is known and is equal to the maximum value $n_{f}^{\max }$ so that only gas saturation remains to be calculated using Eq. (26). On the other hand, within the high-quality regime, one should first determine the gas saturation by solving Eq. (27), and after use Eq. (23) to calculate the corresponding foam texture value.

In practice, for any foam quality value $\left.f_{g} \in\right] 0,1$ [, the solution $S_{g}$ is obtained using the following algorithm:

1. Find the gas saturation by solving the equality (27).

2. Calculate the corresponding foam texture from Eq. (23).

3. If the obtained foam texture is lower than the limiting foam texture $n_{f}^{\max }$, a high-quality regime is obtained, and in this case the resolution process is iterated with another $f_{g}$. Otherwise, a low-quality regime is achieved with a maximum foam texture that is equal to $n_{f}^{\max }$, and the gas saturation has to be determined again from Eq. (26) before proceeding with another $f_{g}$.

This algorithm computes the exact fractional flow of gas in the presence of foam as a function of the gas saturation, hence the foam texture at local equilibrium. However, the uniqueness of the solution $S_{g}$ within the two regimes has to be checked. For this reason, we plot in Fig. 2 the foam textures $n_{f}^{\mathrm{LE}}\left(S_{g}\right)$ and $n_{f}^{\mathrm{FF}}\left(S_{g}\right)$ for several foam qualities and we verify the solution uniqueness. Their intersection yields the gas saturation that corresponds to a given foam texture within the high-quality regime. Intersection of $n_{f}^{\max }$ line and $n_{f}^{\mathrm{FF}}$ yields the gas saturation within the low-quality regime.

Fig. 2 shows that the intersection points between $n_{f}^{\max }$ line and $n_{f}^{\mathrm{FF}}$ on the one hand, and $n_{f}^{\mathrm{LE}}\left(S_{g}\right)$ and $n_{f}^{\mathrm{FF}}\left(S_{g}\right)$ on the other hand, are unique for each foam quality (see also Table 3 which reports a few saturation and texture solutions). Thus, the optimal set of Kam et al model parameters ensure the uniqueness of the solution (foam texture and gas saturation) for both low- and high-quality regimes.

Finally, to check the validity and accuracy of that PB model, the apparent foam viscosity data from Alvarez et al experiments were recalculated using above foam texture and gas saturation solutions. More precisely, for any given value of foam quality, the foam apparent viscosity is recalculated as $\frac{1}{\mu_{f}^{\mathrm{app}}}=\frac{k_{r w}}{\mu_{w}}+\frac{k_{r g}}{\mu_{g}^{\mathrm{PB}}}$ (derived from $u=u_{w}+u_{g}^{f}=\frac{k|\nabla P|}{\mu_{f}^{\text {app }}}=$ $\left.\frac{k k_{r w}|\nabla P|}{\mu_{w}}+\frac{k k_{r g}|\nabla P|}{\mu_{g}^{\mathrm{PB}}}\right)$ where $\mu_{g}^{\mathrm{PB}}=\mu_{g}+\left(\frac{\Phi}{u}\right)^{1 / 3} \frac{c_{f} n_{f}}{\left(f_{g} / S_{g}\right)^{1 / 3}}$ is the effective gas viscosity given by Eq. (5), and where $n_{f}$ and $S_{g}$ are the foam texture and the saturation solutions determined at local equilibrium as before. Table 3 reports the detailed results of this calculation for a few qualities ranging from 0.1 to 0.9 .

Fig. 4 reports the obtained results over the entire foam quality range: one can observe the very good agreement of the computed apparent foam viscosity from Kam et al model with the measurements of Alvarez et al [32], both at the low- and high-quality regimes. Furthermore, the obtained results point out an abrupt foam collapse that occurs at almost a single water saturation $S_{w}^{*-\mathrm{PB}}=0.31$. Thus, a PB model, such as Kam et al model, can be used to properly fit steady state foam mobility at both high-quality and low-quality regimes. 


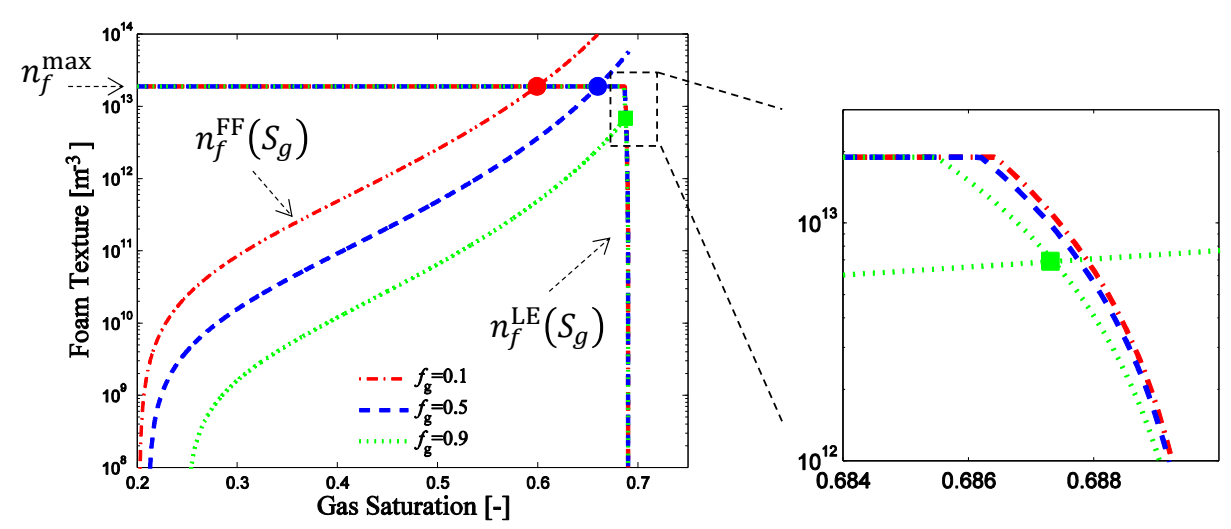

Fig. 2: Derived foam texture at local equilibrium using Kam et al optimal model adjusted by Lotfollahi et al [35] on Alvarez et al experiments [32]. The points corresponding to the solutions for each foam quality are given by circular markers (०) for $n_{f}=n_{f}^{\max }$ and square markers $(\square)$ for $n_{f}<n_{f}^{\max }\left(n_{f}^{\max }=19.1 \times 10^{12} \mathrm{~m}^{-3}\right)$.

Table 3: Steady state foam properties predicted by Kam et al PB model $\left(n_{f}^{\max }=19.1 \times\right.$ $\left.10^{12} \mathrm{~m}^{-3}\right)$

\begin{tabular}{llllll}
\hline$f_{g}(-)$ & $S_{g}(-)$ & $n_{f}\left(\mathrm{~m}^{-3}\right)$ & $n_{D}(-)$ & $\mu_{g}^{\mathrm{PB}}(\mathrm{cP})$ & $\mu_{f}^{\mathrm{app}}(\mathrm{cP})$ \\
\hline 0.1 & 0.5988 & $19.1 \times 10^{12}$ & 1 & $1.7134 \times 10^{3}$ & 310 \\
0.3 & 0.6370 & $19.1 \times 10^{12}$ & 1 & $1.2128 \times 10^{3}$ & 584 \\
0.5 & 0.6595 & $19.1 \times 10^{12}$ & 1 & $1.0348 \times 10^{3}$ & 778 \\
0.7 & 0.6807 & $19.1 \times 10^{12}$ & 1 & $0.9348 \times 10^{3}$ & 928 \\
0.9 & 0.6873 & $6.93 \times 10^{12}$ & 0.36 & $0.3137 \times 10^{3}$ & 393 \\
\hline
\end{tabular}

\subsection{Identification of SE model to calibrated PB model}

The Kam et al PB model, as calibrated and validated before, is used to directly identify the SE model following the procedure of Section 3, with the relationship between fluid saturation and foam texture as determined in Section 4. First, we determine the limiting water saturation $S_{w}^{*-S E}$ by inverting the computed dimensionless foam texture function $n_{D}$. We found that $S_{w}^{*-\mathrm{SE}}=n_{D}^{-1}\left(\frac{1}{2}\right)=0.313$. In addition, the parameter $\Theta$ is calculated ensuring $F_{2}\left(S_{w}^{*-\mathrm{PB}}\right)=\varepsilon$. A tolerance $\varepsilon$ of 0.05 is applied in order to render at best the dimensionless foam texture variation, leading to a value of $\Theta=2.1 \times 10^{3}$, as reported in Table 4. Fig. 3(a) shows the resulting dry-out function $F_{2}$ and the dimensionless foam texture function $n_{D}$ as functions of water saturation.

The reference capillary number, given by Eq. (11), is set at the lowest reached value during the experiments, namely $N_{c g}^{\mathrm{ref}}=\left(\mu_{g} f_{g}^{\mathrm{min}} u\right) /\left(\sigma \Phi S_{g}^{\mathrm{min}}\right)$ where $f_{g}^{\mathrm{min}}=0.25$ is the lowest foam quality. The lowest gas saturation $S_{g}^{\min }$ is calculated as follows. By application of the generalized Darcy law to the water phase one has $u_{w}=\frac{k k_{r w}}{\mu_{w}}|\nabla P|$ hence $k_{r w}=\frac{\mu_{w} u f_{w}}{k|\nabla P|}$. With a $k_{r w}$ power law function such that $k_{r w}=k_{r w}^{0} S^{n_{w}}$ with $S=\frac{S_{w}-S_{w i}}{1-S_{g r}-S_{w i}}, S_{w}$ can be written as 
$S_{w}=S_{w i}+\left(1-S_{w i}-S_{g r}\right)\left(\frac{k_{r w}}{k_{r w}^{w}}\right)^{1 / n_{w}}$. Replacing $k_{r w}$ by the above expression versus $|\nabla P|$ we obtain $S_{w}=S_{w i}+\left(1-S_{g r}-S_{w i}\right)\left(\frac{\mu_{w} u f_{w}}{k|\nabla P| k_{r w}^{0}}\right)^{1 / n_{w}}$. Applying the one-phase Darcy law to the equivalent fluid yields $\mu_{f}^{\text {app }}=\frac{k|\nabla P|}{u}$, and as a consequence

$$
S_{w}=S_{w i}+\left(1-S_{w i}-S_{g r}\right)\left(\frac{\mu_{w}\left(1-f_{g}\right)}{\mu_{f}^{\mathrm{app}} k_{r w}^{0}}\right)^{1 / n_{w}} .
$$

Therefore, the lowest gas saturation $S_{g}^{\min }$ can be calculated as $S_{g}^{\min }=1-S_{w i}-(1-$ $\left.S_{g r}-S_{w i}\right)\left(\frac{\mu_{w}\left(1-f_{g}^{\min }\right)}{\mu_{f}^{\text {ap }} k_{r w}^{0}}\right)^{1 / n_{w}}$. Specifically, one has $f_{g}^{\min }=0.25$ and $S_{g}^{\text {min }}=0.63$ for Alvarez et al measurements, hence $N_{c g}^{\text {ref }}=1.27 \times 10^{-8}$, as reported in Table 4. The obtained shearthinning function $F_{4}$ is reported in Fig. 3(b). Note that according to this identification method, the $N_{c g}^{\text {ref }}$ value does not affect the SE model results as $M_{\text {ref }}$ and $N_{c g}^{\text {ref }}$ are correlated as given by Eq. (20).

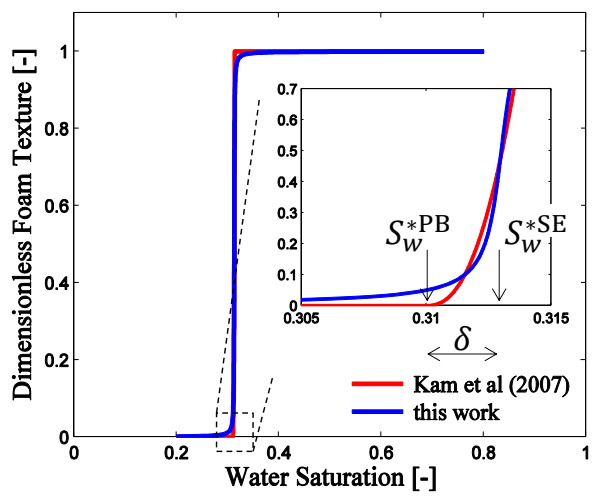

(a)

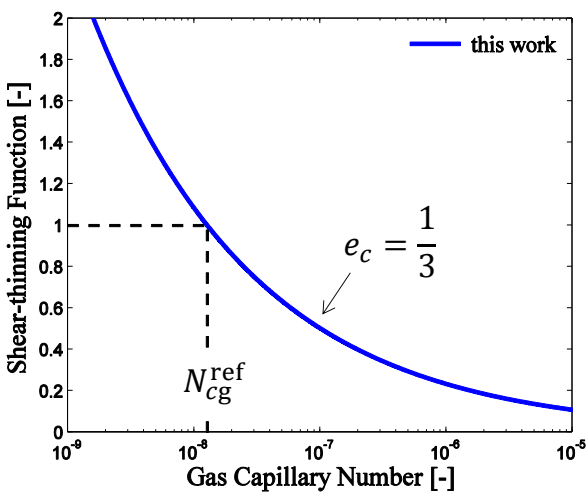

(b)

Fig. 3: (a) Dimensionless foam texture obtained explicitly from Kam et al model and implicitly from our model to fit Alvarez et al measurements; (b) Shear-thinning function $F_{4}$ used in our SE model.

The SE model parameters, which are reported in Table 4, are then used to calculate the apparent foam viscosity $\mu_{f}^{\text {app }}$ which can be written in the SE model framework as $\frac{1}{\mu_{f}^{\text {app }}}=$ $\frac{k_{r w}}{\mu_{w}}+\frac{F M \cdot k_{r g}}{\mu_{g}}$, derived again from the expression of total velocity as the sum of water and gas velocities, with gas velocity from Eq. (8) where $F M$ is given by Eq. (9), and only involves the product of $F_{2}$ and $F_{4}$ functions. The water saturation corresponding to each foam quality is obtained by solving the gas fractional flow equation $f_{g}=1 /\left(1+\frac{\mu_{g}}{\mu_{w}} \frac{k_{r w}}{F M \cdot k_{r g}}\right)$. Table 5 reports the detailed results of this calculation for a few qualities ranging from 0.1 to 0.9 . The so obtained apparent foam viscosity, which is reported in Fig. 4(a), is very close to the measured one and to the one derived from the Kam PB model over the entire foam quality interval.

In practice, the SE parameters would be used to simulate the displacement for several foam qualities. The simulated steady state pressure gradient $|\nabla P|=\frac{\Delta P}{L}$ allows to compute 
Table 4: SE model parameters directly estimated from the calibrated PB model.

\begin{tabular}{lllll}
\hline$M_{\text {ref }}(-)$ & $S_{w}^{*-S E}(-)$ & $\Theta(-)$ & $N_{c g}^{\mathrm{ref}}(-)$ & $e_{c}(-)$ \\
\hline $6.4637 \times 10^{4}$ & 0.313 & $2.1 \times 10^{3}$ & $1.27 \times 10^{-8}$ & $\frac{1}{3}$ \\
\hline
\end{tabular}

Table 5: Steady state foam properties predicted by our SE model.

\begin{tabular}{llllll}
\hline$f_{g}(-)$ & $S_{w}(-)$ & $F_{2}(-)$ & $F_{4}(-)$ & $F M(-)$ & $\mu_{f}^{\mathrm{app}}(\mathrm{cP})$ \\
\hline 0.1 & 0.4013 & 0.9983 & 1.3254 & $0.1169 \times 10^{-4}$ & 309 \\
0.3 & 0.3631 & 0.9970 & 0.9381 & $0.1654 \times 10^{-4}$ & 582 \\
0.5 & 0.3407 & 0.9945 & 0.8004 & $0.1944 \times 10^{-4}$ & 774 \\
0.7 & 0.3199 & 0.9780 & 0.7229 & $0.2188 \times 10^{-4}$ & 909 \\
0.9 & 0.3128 & 0.3620 & 0.6671 & $0.6407 \times 10^{-4}$ & 391 \\
\hline
\end{tabular}

the foam apparent viscosity $\mu_{f}^{\text {app }}=\frac{k|\nabla P|}{u}$ by application of the one-phase Darcy law to the equivalent fluid. Table 6 reports the so obtained apparent foam viscosity derived from the simulated pressure gradient for several qualities, with our SE model. As expected, these simulated values are very close to the ones reported in Table 5, since most of the relative errors with respect to the reported values in Table 5 are of the order of $1 \%$.

Table 6: Apparent foam viscosity, for several qualities, predicted by our SE model from the simulated steady state pressure gradient $|\nabla P|=\frac{\Delta P}{L}$ across the core. Relative errors $\Delta_{S_{w}}$ and $\Delta_{\mu_{f}}$ app refer to steady state values reported in Table 5 .

\begin{tabular}{lllllll}
\hline$f_{g}(-)$ & $S_{w}(-)$ & $\Delta_{S_{w}}(\%)$ & $\Delta P($ bar $)$ & $|\nabla P|($ bar $/ \mathrm{m})$ & $\mu_{f}^{\text {app }}(\mathrm{cP})$ & $\Delta_{\mu_{f}^{\text {app }}(\%)}$ \\
\hline 0.1 & 0.3942 & 1.8 & 14.3345 & 51.2460 & 303.93 & 1.6 \\
0.3 & 0.3571 & 1.7 & 27.1031 & 96.8940 & 574.66 & 1.3 \\
0.5 & 0.3355 & 1.5 & 36.0873 & 129.0124 & 765.15 & 1.1 \\
0.7 & 0.3161 & 1.2 & 41.4113 & 148.0458 & 878.03 & 3.4 \\
0.9 & 0.3118 & 0.3 & 19.1824 & 68.5774 & 406.72 & 4.0 \\
\hline
\end{tabular}

The apparent foam viscosities computed from SE model and PB model at local equilibrium are almost equal over the whole range of foam qualities. The maximum apparent foam viscosity and the optimal foam quality are also successfully predicted by the SE model. We note a slightly smoother transition between low- and high-quality regimes than the sharp transition predicted by Kam et al model. A larger value of $\Theta$ parameter could have been used to refine the fit in the transition region around the optimal foam quality $f_{g}^{*}$.

Table 7 reports the so obtained PB and SE models apparent foam viscosities and their relative errors with respect to the measurements $\Delta_{\mathrm{PB}}=\left|\left(\mu_{f}^{\mathrm{app}}\right)_{\mathrm{PB}}-\mu_{f}^{\mathrm{app}}\right| / \mu_{f}^{\mathrm{app}}$ and $\Delta_{\mathrm{SE}}=$ $\left|\left(\mu_{f}^{\text {app }}\right)_{\mathrm{SE}}-\mu_{f}^{\mathrm{app}}\right| / \mu_{f}^{\mathrm{app}}$. These relative errors are also reported in Fig. 4(b). Overall, they do not exceed $5 \%$ except for large qualities. Thus, both models adjust equally well the steady state measurements of the apparent foam viscosity.

To conclude, the main input for SE model identification to PB model is the relationship between fluid saturation and the foam texture predicted by PB model. As expected, the 


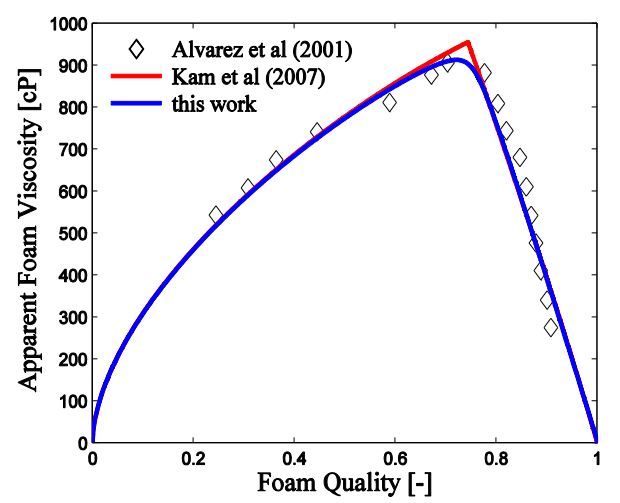

(a)

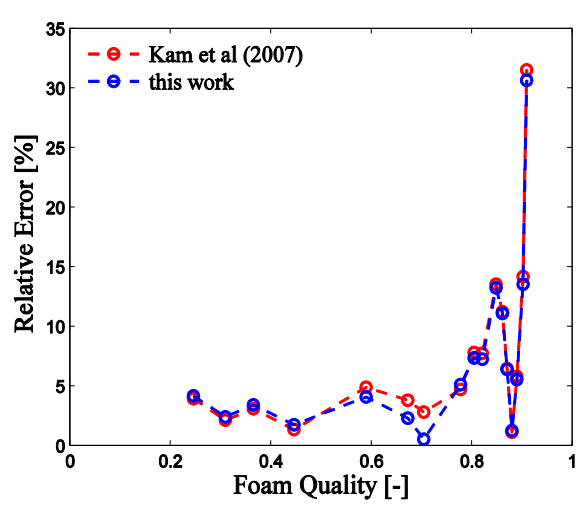

(b)

Fig. 4: (a) Comparison of Alvarez et al apparent foam viscosity measurements with the apparent viscosity computed from SE model with parameters directly estimated from the calibrated Kam et al model; (b) Apparent foam viscosity relative errors with respect to the measurements.

Table 7: Apparent foam viscosities and relative errors with respect to the measurements, both for Kam PB model $\left(\Delta_{\mathrm{PB}}\right)$ and our SE model $\left(\Delta_{\mathrm{SE}}\right.$; see the text).

\begin{tabular}{lllllll}
\hline & \multicolumn{3}{c}{ Measurements } & \multicolumn{2}{c}{ Kam et al PB model } & \multicolumn{2}{c}{ Our SE model } \\
& $f_{g}(-)$ & $\mu_{f}^{\text {app }}(\mathrm{cP})$ & $\mu_{f}^{\mathrm{app}}(\mathrm{cP})$ & $\Delta_{\mathrm{PB}}(\%)$ & $\mu_{f}^{\text {app }}(\mathrm{cP})$ & $\Delta_{\mathrm{SE}}(\%)$ \\
\hline & 0.25 & 542.2 & 520.8 & 4 & 519.5 & 4 \\
& 0.30 & 607.4 & 594.3 & 2 & 592.6 & 2 \\
Low-quality & 0.36 & 674.0 & 653.0 & 3 & 650.9 & 3 \\
regime & 0.45 & 740.7 & 730.7 & 1 & 727.6 & 2 \\
& 0.59 & 810.6 & 850.4 & 5 & 843.9 & 4 \\
& 0.67 & 876.5 & 910.1 & 4 & 896.9 & 2 \\
& 0.70 & 905.6 & 931.1 & 3 & 910.6 & 1 \\
\hline & 0.78 & 881.4 & 839.7 & 5 & 836.2 & 5 \\
& 0.80 & 808.6 & 745.3 & 8 & 749.0 & 7 \\
High-quality & 0.82 & 743.7 & 685.9 & 8 & 689.6 & 7 \\
regime & 0.85 & 680.0 & 587.7 & 14 & 589.9 & 13 \\
& 0.86 & 609.8 & 541.2 & 11 & 542.0 & 11 \\
& 0.87 & 541.7 & 506.6 & 6 & 506.9 & 6 \\
& 0.88 & 476.1 & 470.6 & 1 & 470.0 & 1 \\
& 0.89 & 410.2 & 434.1 & 6 & 433.0 & 6 \\
& 0.90 & 339.7 & 387.9 & 14 & 385.8 & 14 \\
& 0.91 & 273.9 & 360.3 & 32 & 357.9 & 31 \\
\hline
\end{tabular}

resulting SE model is found to fit foam quality-scan experiments as well as the original PB model. This opens the way for reliable use of foam flow models for reservoir applications. 


\subsection{Comparison with other calibration methods}

Several adjustment procedures of the apparent foam viscosity measurements to a single scan of foam quality are available in the literature. They are reviewed and discussed hereafter.

Boejie and Rossen [21] proposed a simple manual procedure to fit SE models to the apparent foam viscosity measurements. The procedure is based on six steps dealing separately with the data in the high-quality and low-quality regimes. The latter approach assumes an abrupt transition between the two regimes such that large values of $\Theta$ are considered beforehand $\left(\Theta\right.$ between $10^{4}$ and $\left.10^{5}\right)$. In addition, the pressure gradient is assumed to decrease linearly with the foam quality, in the high-quality regime. The other SE model parameters are calculated step by step. The proposed approach is simple as it can be carried out directly from the plot of the apparent foam viscosity versus foam quality. Nevertheless, the method cannot fit properly the measurements when the experimental data show a gradual transition between high- and low-quality regimes. In that case, the authors propose to use the results of their method to initiate a least squares fit of all foam model parameters to further tune the SE model.

Lotfollahi et al [35] used a least squares minimization method to fit different SE models and PB models to two experimental data sets from Alvarez et al [32] and Moradi-Araghi et al [33]. The fit is performed within the two regimes using all the measurements. The SE model parameters to be optimized are $M_{\text {ref }}, S_{w}^{*-\mathrm{SE}}, \Theta$ and $e_{c}$. The authors indicate that the final fit may depend on the initial guess, that is the method does not guarantee to yield the optimal model. This non-uniqueness is also pointed out by Kapetas et al [36], who obtained different sets of foam parameters when applying the two previous methods on a large set of experimental data.

To improve the model match, Farajzadeh et al [12] used data weighting and constraints in the least squares minimization method. In addition, they added liquid saturation values for each measurement to the definition of the objective function which reads in this case $\mathscr{O}(X)=$ $\frac{1}{2} \sum_{j=1}^{n_{\text {data }}} w_{j}\left[\mu_{f, j}^{\text {app }}\left(X, S_{w, j}\right)-\mu_{f, j}^{\exp }\right]^{2}$, where $X=\left(M_{\text {ref }}, S_{w}^{*-S E}, \Theta, e_{c}\right)$ is vector of parameters to be optimized, $w_{j}$ weights, $\mu_{f, j}^{\text {app }}$ computed apparent foam viscosity, $\mu_{f, j}^{\exp }$ measured apparent foam viscosity and $S_{w, j}$ is liquid saturation for each data point which is calculated from the liquid relative permeability as $S_{w, j}=S_{w i}+\left(1-S_{g r}-S_{w i}\right)\left(\frac{\mu_{w}\left(1-f_{g, j}\right)}{\mu_{f, j}^{\exp } k_{r w}^{0}}\right)^{1 / n_{w}}$ for power law $k_{r}$ functions, as explained in Section 5.3 [see Eq. (28)].

Assuming no shear-thinning effect in foam quality-scan experiments, Ma et al [37] proposed a graphical method with $M_{\text {ref }}$ and $S_{w}^{*-S E}$ as variables, in order to match the transition foam viscosity between the low- and high-quality regimes. The $\Theta$ parameter is matched after with a least squares method applied to the other steady state apparent viscosity measurements. Later on, Ma et al [20] determined the same three model parameters $\left(M_{\mathrm{ref}}, S_{w}^{*-\mathrm{SE}}\right.$, $\Theta)$ by using a least squares minimization method as other authors, but adding a constraint on $S_{w}^{*-S E}$ to avoid non unique $\left(M_{\text {ref }}, S_{w}^{*-S E}\right)$ solutions.

Then, Zeng et al [38] converted the three-parameter $\left(M_{\mathrm{ref}}, S_{w}^{*-\mathrm{SE}}, \Theta\right)$ optimization problem (Ma et al [20]) to a single-parameter $\left(M_{\text {ref }}\right)$ problem by using steady state apparent viscosity data and searching the $M_{\text {ref }}$ value that fits a linear function of the saturation with $\left(S_{w}^{*-S E}, \Theta\right)$ as parameters. The same authors also adjusted the shear-thinning function separately from total-flow-rate-scan experiments.

To end with, this limited survey of SE models indicate that calibration methods are based on a direct identification of the parameters of the SE model relative permeability function, without using the physical representation of foam as a succession of lamellas. Many alternative methods of implementation have been developed to deal with the multi- 
variable nature of the identification problem and with the peculiarity of foam flow equations involving discontinuities in solutions. The method of this paper aims at overcoming these difficulties via the PB model representation as flow-restricting lamellas.

\section{Conclusions and perspectives}

The objective of this paper was to establish the equivalence between semi-empirical foam flow models, based on a modified relative permeability concept, and population-balance models that keeps track of the intrinsic physics of foam that explains mobility reduction in porous media. The identification of both foam flow models leads to a method for calibrating SE models from the PB model translation (i.e. foam-texture translation) of foam flow data. This physical approach of foam flow could circumvent some difficulties in the direct calibration of SE model from foam mobility (or apparent viscosity) data. The use of least squares method is not really adapted to the calculation of a flow model involving discontinuities as observed with foams. Lamellas-based models involve the foam texture physical variable whose evolution is precisely responsible of more or less pronounced foam flow discontinuities. The method developed and discussed in this paper calls for further developments regarding the impact of capillary pressure on the foam SE model parameters. In addition, the question has to be addressed for reservoir applications involving different rock permeabilities and pore structures.

\section{References}

1. D. G. Bond and O. C. Holbrook. Gas drive oil recovery. Patent no. US Pat 2866507, 1958.

2. L. W. Holm. Foam injection test in the Siggins field, Illinois. Society of Petroleum Engineers, $22,1970$.

3. T. M. Jonas, S. I. Chou, and S. L. Vasicek. Evaluation of a $\mathrm{CO}_{2}$ foam field trial: Rangely Weber Sand Unit. Society of Petroleum Engineers, Paper SPE-20468-MS, 1990.

4. R. J. Watts J. P. Heller, D. A. Boone. Field test of $\mathrm{CO}_{2}$ mobility control at Rock Creek. Society of Petroleum Engineers, Paper SPE-14395-MS, 1995.

5. A. H. Falls, G. J. Hirasaki, T. W. Patzek, D. A. Gauglitz, D. D. Miller, and T. Ratulowski. Development of a mechanistic foam simulator: the population balance and generation by snap-off. SPE Reservoir Engineering, 3(3):884-892, 1988.

6. A. R. Kovscek and C. J. Radke. Fundamentals of foam transport in porous media. in Foams: fundamentals and applications in the petroleum industry, American Chemical Society Advances in Chemistry (M. J. Comstock and L. L. Schramm Eds), 1994.

7. G. J. Hirasaki and J. B. Lawson. Mechanisms of foam flow in porous media: apparent viscosity in smooth capillaries. SPE Journal, 25(2):176-190, 1985.

8. F. P. Bretherton. The motion of long bubbles in tubes. Journal of Fluid Mechanics, 10(2):166, 1961.

9. T. C. Ransohoff and C. J. Radke. Mechanisms of foam generation in glass bead packs. SPE Reservoir Engineering, 3:573-585, 1988.

10. Z. I. Khatib, G. J. Hirasaki, and A. H. Falls. Effects of capillary pressure on coalescence and phase mobilities in foams flowing through porous media. SPE Reservoir Engineering, 3(3):919-926, 1988.

11. K. T. Chambers and C. J. Radke. Capillary phenomena in foam flow through porous media. Interfacial Phenomena in Petroleum Recovery, N. R. Morrow (ed.) (Marcel Dekker, Inc), 1991.

12. R. Farajzadeh, M. Lotfollahi, A. A. Eftekhari, W. R. Rossen, and G. J. H. Hirasaki. Effect of permeability on implicit-texture foam model parameters and the limiting capillary pressure. Energy \& Fuels, 29(5):3011-3018, 2015.

13. W. T. Osterloh and M. J. Jante. Effects of gas and liquid velocity on steady-state foam flow at high temperature. SPE/DOE 24179, 1992.

14. F. Friedmann, W. H. Chen, and P. A. Gauglitz. Experimental and simulation study of high-temperature foam displacement in porous media. SPE Reservoir Engineering, 6(1):37-45, 1991.

15. R. A. Ettinger and C. J. Radke. Influence of texture on steady foam flow in Berea sandstone. $S P E$ Reservoir Engineering, 7(1):83-90, 1992. 
16. A. R. Kovscek and H. J. Bertin. Foam mobility in heterogeneous porous media. Transport in Porous Media, 52(1):37-49, 2003.

17. Q. Chen, A. R. Kovscek, and M. Gerritsen. Modeling foam displacement with the local-equilibrium approximation: theory and experimental verification. SPE Journal, 15(01):171-183, 2010.

18. H. Martinsen and F. Vassenden. Foam assisted water alternating gas (FAWAG) process on Snorre. $10^{\text {th }}$ European Symposium on Improved Oil Recovery, Brighton, UK, 1999.

19. L. Cheng, A. B. Reme, D. Shan, D. A. Coombe, and W. R. Rossen. Simulating foam processes at high and low foam qualities. SPE/DOE Improved Oil Recovery Symposium, Tulsa, Oklahoma, 2000.

20. K. Ma, R. Farajzadeh, J. L. Lopez-Salinas, C. A. Miller, S. L. Biswal, and G. J. Hirasaki. Nonuniqueness, numerical artifacts, and parameter sensitivity in simulating steady-state and transient foam flow through porous media. Transport in Porous Media, 102(3):325-348, 2014.

21. C. S. Boeije and W. R. Rossen. Fitting foam-simulation-model parameters to data: I. coinjection of gas and liquid. SPE Reservoir Evaluation \& Engineering, 18(2):264-272.

22. G. Bernard and W. L. Jacobs. Effect of foam on trapped gas saturation and on permeability of porous media to water. SPE Journal, 5(4):295-300, 1965.

23. J. B. Lawson and J. Reisberg. Alternate slugs of gas and dilute surfactant for mobility control during chemical flooding. SPE/DOE Enhanced Oil Recovery Symposium, Tulsa, Oklahoma, 1980.

24. D. W. Peaceman. Fundamentals of numerical reservoir simulation, volume 6 of Developments in Petroleum Science. Elsevier Science, Amsterdam, 1977.

25. J. A. Trangenstein and J. B. Bell. Mathematical structure of the black-oil model for petroleum reservoir simulation. SIAM J. Appl. Math., 49(3):749-783, 1989.

26. L. W. Lake. Enhanced oil recovery. Prentice Hall, 1989.

27. D. W. Green and G. P. Willhite. Enhanced oil recovery, volume 6 of SPE Textbook Series. Henry L. Doherty Memorial Fund of AIME, Society of Petroleum Engineers, 1998.

28. C. M. Marle. Multiphase flow in porous media. Gulf Publishing Company, third edition, 1981.

29. T. W. Patzek. Description of foam flow in porous media by the population balance method. in Surfactantbased mobility control, American Chemical Society Symposium Series (H. S. Duane Ed.), 1988.

30. S. I. Kam, Q. P. Nguyen, Q. Li, and W. R. Rossen. Dynamic simulations with an improved model for foam generation. SPE Journal, 12(1):35-48, 2007.

31. S. I. Kam. Improved mechanistic foam simulation with foam catastrophe theory. Colloids and Surfaces A: Physicochemical and Engineering Aspects, 318(1-3):62-77, 2008.

32. J. M. Alvarez, H. J. Rivas, and W. R. Rossen. Unified model for steady-state foam behavior at high and low foam qualities. SPE Journal, 6(3):325-333, 2001.

33. A. Moradi-Araghi, E. L. Johnston, D. R. Zornes, and K. J. Harpole. Laboratory evaluation of surfactants for $\mathrm{CO}_{2}$-foam applications at the South Cowden Unit. International Symposium on Oilfield Chemistry, Houston, Texas, 1997.

34. B. Braconnier, E. Flauraud, and Q. L. Nguyen. Efficient scheme for chemical flooding. Oil \& Gas Science and Technology - Rev. IFP Nouvelles, 69(4):585-601, 2014.

35. M. Lotfollahi, R. Farajzadeh, M. Delshad, A. Varavei, and W. R. Rossen. Comparison of implicit-texture and population-balance foam models. Journal of Natural Gas Science and Engineering, 31:184-197, 2016.

36. L. Kapetas, S. Vincent-Bonnieu, R. Farajzadeh, A. A. Eftekhari, S. R. Mohd-Shafian, R. Z. Kamarul Bahrim, and W. R. Rossen. Effect of permeability on foam-model parameters - an integrated approach from coreflood experiments through to foam diversion calculations. $18^{\text {th }}$ European Symposium on Improved Oil Recovery, Dresden, 14-16 April, 2015.

37. K. Ma, J. L. Lopez-Salinas, M. C. Puerto, C. A. Miller, S. L. Biswal, and G. J. Hirasaki. Estimation of parameters for the simulation of foam flow through porous media. part 1: the dry-out effect. Energy \& Fuels, 27(5):2363-2375, 2013.

38. Y. Zeng, A. Muthuswamy, K. Ma, W. Le, R. Farajzadeh, M. Puerto, S. Vincent-Bonnieu, A. A. Eftekhari, Y. Wang, C. Da, J. C. Joyce, S. L. Biswal, and G. J. Hirasaki. Insights on foam transport from a textureimplicit local-equilibrium model with an improved parameter estimation algorithm. Industrial \& Engineering Chemistry Research, 55(28):7819-7829, 2016. 